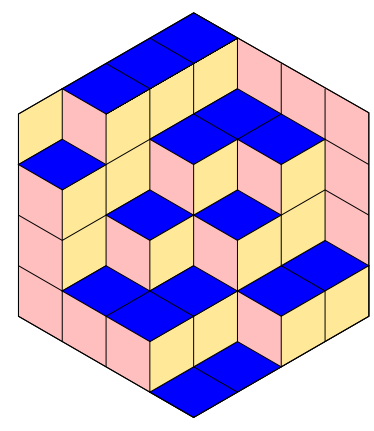

ALGEBRAIC COMBINATORICS

Aldo Conca, Emanuela De Negri \& Željka Stojanac

A characteristic free approach to secant varieties of triple Segre products

Volume 3, issue 5 (2020), p. 1011-1021.

<http://alco.centre-mersenne.org/item/ALCO_2020__3_5_1011_0>

(C) The journal and the authors, 2020.

Some rights reserved.

(c) BY This article is licensed under the

Creative Commons ATtribution 4.0 International LiCEnSE.

http://creativecommons.org/licenses/by/4.0/

Access to articles published by the journal Algebraic Combinatorics on the website http://alco.centre-mersenne.org/ implies agreement with the Terms of Use (http://alco.centre-mersenne.org/legal/).

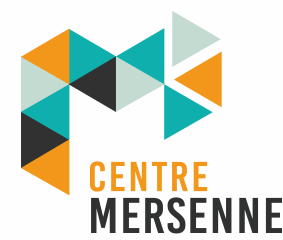

Algebraic Combinatorics is member of the Centre Mersenne for Open Scientific Publishing www.centre-mersenne.org 


\title{
A characteristic free approach to secant varieties of triple Segre products
}

\author{
Aldo Conca, Emanuela De Negri \& Željka Stojanac
}

\begin{abstract}
The goal of this short note is to study the secant varieties of the Segre embedding of the product $\mathbb{P}^{1} \times \mathbb{P}^{a-1} \times \mathbb{P}^{b-1}$ by means of the standard tools of combinatorial commutative algebra. We reprove and extend to arbitrary characteristic the results of Landsberg and Weyman [5] regarding the defining ideal and the Cohen-Macaulay property of the secant varieties. Furthermore we compute their degrees and give a bound for their Castelnuovo-Mumford regularities, which are sharp in many cases.
\end{abstract}

\section{INTRODUCTION}

The topic of this paper is the secant varieties of the triple Segre product of $\mathbb{P}^{1} \times$ $\mathbb{P}^{a-1} \times \mathbb{P}^{b-1}$. For basic facts about tensor decomposition and Cohen-Macaulayness of secant varieties of Segre products we refer the reader to Oeding's paper [7] or to the book of Landsberg [4]. We will work with 3 -tensors of size $(2, a, b)$ with $2 \leqslant a \leqslant b$. The variety of rank-1 tensors is denoted by $\operatorname{Seg}(2, a, b)$ and corresponds to the image of the Segre embedding of $\mathbb{P}^{1} \times \mathbb{P}^{a-1} \times \mathbb{P}^{b-1}$ in $\mathbb{P}^{2 a b-1}$. We will further denote by $\sigma_{t}(2, a, b)$ the $t$-secant variety of $\operatorname{Seg}(2, a, b)$, that is, the variety of rank- $t$ tensors. The defining ideal of $\operatorname{Seg}(2, a, b)$ in $R=K\left[x_{i j k}:(i, j, k) \in[2] \times[a] \times[b]\right]$ will be denoted by $I(a, b)$. The ideal defining the secant variety $\sigma_{t}(2, a, b)$ will be denoted by $I(a, b)^{\{t\}}$.

We recall that $\operatorname{Seg}(2, a, b)$ is a well understood toric variety whose defining ideal is the Hibi ideal of $[2] \times[a] \times[b]$. In other words, equipping $[2] \times[a] \times[b]$ with the natural structure of distributive lattice one has:

$$
I(a, b)=\left(x_{\alpha} x_{\beta}-x_{\alpha \wedge \beta} x_{\alpha \vee \beta}: \alpha, \beta \text { are incomparable elements of }[2] \times[a] \times[b]\right) .
$$

Our goal it to prove that the ideal $I(a, b)^{\{t\}}$ is generated by determinantal equations coming from "unfoldings" of the associated tensors and that it defines a CohenMacaulay ring. These results are already known in characteristic 0 , see [5], while our results are valid in arbitrary characteristic. Furthermore our construction allows us to give a formula for the degree of the secant varieties $\sigma_{t}(2, a, b)$, and to give a bound for their Castelnuovo-Mumford regularity; this bound is sharp if $2 t \leqslant b$. We also

\footnotetext{
Manuscript received 29th July 2019, revised 10th February 2020, accepted 12th February 2020.
}

KEYWORDS. Segre products, secant varieties, Gröbner bases, tensors.

ACKnowledgements. A. Conca and E. De Negri were partially supported by INDAM. Z. Stojanac has been supported by the Excellence Initiative of the German Federal and State Governments (Grant 81), the DFG projects GRO 4334/1,2 (SPP1798 CoSIP) and by INdAM during her one-month visit to the Department of Mathematics of the University of Genova in the spring of 2017. 
conjecture that $I(2 t, 2 t)^{\{t\}}$ defines a Gorenstein ring for all $t \geqslant 1$ and check it for $t \leqslant 3$.

The unfolding (a.k.a. flattening) is a transformation that reorganizes a tensor into a matrix. For a tensor of size $n_{1} \times n_{2} \times \cdots \times n_{d}$, the $k$-th unfolding is a matrix of size $n_{k} \times\left(n_{1} \cdots n_{k-1} n_{k+1} \cdots n_{d}\right)$. The rows are indexed by the $k$-th index, and the columns are indexed by the vectors of the remaining indices. The order on the row and column indices is not important. In the following we will order them lexicographically.

ExAmple 1.1. Let $\mathcal{X}$ be an order-3 tensor of size $2 \times 3 \times 3$. Then, the first, second, and third unfolding are of the form

$$
\begin{aligned}
& \mathcal{X}^{\{1\}}=\left[\begin{array}{lllllllll}
x_{111} & x_{112} & x_{113} & x_{121} & x_{122} & x_{123} & x_{131} & x_{132} & x_{133} \\
x_{211} & x_{212} & x_{213} & x_{221} & x_{222} & x_{223} & x_{231} & x_{232} & x_{233}
\end{array}\right] \\
& \mathcal{X}^{\{2\}}=\left[\begin{array}{llllll}
x_{111} & x_{112} & x_{113} & x_{211} & x_{212} & x_{213} \\
x_{121} & x_{122} & x_{123} & x_{221} & x_{222} & x_{223} \\
x_{131} & x_{132} & x_{133} & x_{231} & x_{232} & x_{233}
\end{array}\right] \\
& \mathcal{X}^{\{3\}}=\left[\begin{array}{llllll}
x_{111} & x_{121} & x_{131} & x_{211} & x_{221} & x_{231} \\
x_{112} & x_{122} & x_{132} & x_{212} & x_{222} & x_{232} \\
x_{113} & x_{123} & x_{133} & x_{213} & x_{223} & x_{233}
\end{array}\right] .
\end{aligned}
$$

Set $X=\left[x_{i j}\right], Y=\left[y_{i j}\right]$ with $x_{i j}=x_{1 i j}, y_{i j}=x_{2 i j}$ for all $i \in[a], j \in[b]$. We remark that for $\mathcal{X}$ of size $2 \times a \times b$ the second and the third unfolding of $\mathcal{X}$ are the block matrices

$$
\mathcal{X}^{\{2\}}=[X Y] \quad \text { and } \quad \mathcal{X}^{\{3\}}=\left[X^{T} Y^{T}\right] .
$$

They can be combined in the single arrangement:

$$
\begin{aligned}
& X Y \\
& Y
\end{aligned} .
$$

The $(t+1)$-minors of the various unfolding matrices are contained in the ideal defining the $t$-secant variety of the Segre variety but, in general, one needs extra generators. On the other hand we will see that for the tensor of size $(2, a, b)$ the $(t+1)$-minors coming from the unfolding matrices are enough to generate $I(a, b)^{\{t\}}$.

In the following for a matrix $M$ with entries in a ring $R$ and $t \in \mathbb{N}$ we will denote by $I_{t}(M)$ the ideal of $R$ generated by all the $t$-minors of $M$.

\section{GRÖBner bases AND SECANT IDEALS}

In this section we quickly recall the results of Sturmfels and Sullivant [9] and of Simis and Ulrich [8] that we will use.

DeFINITION 2.1. Let $J_{1}, J_{2}, \ldots, J_{r}$ be ideals in a polynomial ring $R=K\left[x_{1}, x_{2}, \ldots, x_{n}\right]$ over a field $K$. Their join $J_{1} * J_{2} * \cdots * J_{r}$ is the elimination ideal in $R$

$$
\left(J_{1}\left(\mathbf{y}_{1}\right)+\cdots+J_{r}\left(\mathbf{y}_{r}\right)+\left(y_{1 i}+y_{2 i}+\cdots+y_{r i}-x_{i}: 1 \leqslant 1 \leqslant n\right)\right) \cap R,
$$

where $\mathbf{y}_{i}=\left(y_{1 i}, y_{2 i}, \ldots, y_{n i}\right)$ are new indeterminates for all $i \in[r]$ and $J_{i}\left(\mathbf{y}_{i}\right)$ is the image of $J_{i}$ in $K[\mathbf{x}, \mathbf{y}]=K\left[x_{1}, x_{2}, \ldots, x_{n}, y_{11}, y_{12}, \ldots, y_{r n}\right]$ under the map $\mathbf{x} \mapsto \mathbf{y}_{j}$.

We denote by $I^{\{r\}}$ the $r$-fold join of I with itself, that is,

$$
I^{\{r\}}=I * I * \cdots * I .
$$

If $K$ is algebraically closed and $I$ defines an irreducible variety $\mathbb{X} \subset \mathbb{P}^{N}$, then $I^{\{r\}}$ is the defining ideal of the $r$ th secant variety $\mathbb{X}^{\{r\}}$ of $\mathbb{X}$, defined as:

$$
\mathbb{X}^{\{r\}}=\overline{\bigcup_{P_{1}, \ldots, P_{r} \in \mathbb{X}}\left\langle P_{1}, \ldots, P_{r}\right\rangle}
$$


where $\left\langle P_{1}, \ldots, P_{r}\right\rangle$ denotes the linear span of the points $P_{1}, \ldots, P_{r}$ and the bar denotes the Zariski closure.

Let $\Delta$ be a simplicial complex and denote by $I_{\Delta}$ the associated Stanley-Reisner ideal. The ideal $I_{\Delta}^{\{r\}}$ turns out to be square-free, that is,

$$
I_{\Delta}^{\{r\}}=I_{\Delta\{r\}}
$$

where the complex $\Delta^{\{r\}}$ is described as follows:

Proposition 2.2 ([9, Remark 2.9$])$. Every face of $\Delta^{\{r\}}$ is the union of $r$ faces of $\Delta$. Thus if $F_{1}, F_{2}, \ldots, F_{v}$ are the facets of $\Delta$, then the facets of $\Delta^{\{r\}}$ are the maximal subsets of the form $F_{i_{1}} \cup F_{i_{2}} \cup \cdots \cup F_{i_{r}}$ with $1 \leqslant i_{1}<\cdots<i_{r} \leqslant v$.

Moreover the $r$-fold join of an initial ideal contains the initial ideal of $r$-fold join ideal.

Theorem 2.3 ([9, Corollary 4.2]). Let I be an ideal and $\prec$ a term oder. Then one has:

for all $r$.

$$
\operatorname{in}\left(I^{\{r\}}\right) \subseteq \operatorname{in}(I)^{\{r\}}
$$

If the equality holds for every $r$ then the term order is said to be delightful for the ideal $I$.

\section{The IDEAL OF TWO Minors AND THE ASSOCIATED SIMPLiCIAL COMPLEX}

Let $K$ be a field and let $\mathcal{X}=\left(x_{i j k}\right)$ be the tensor of indeterminates of size $2 \times a \times b$, with $2 \leqslant a \leqslant b$.

Let $R=K\left[x_{111}, x_{112}, \ldots, x_{2 a b}\right]$ be the polynomial ring with indeterminates the entries of $\mathcal{X}$. From now on we denote by $\tau$ any diagonal term order, that is, a term order such that the initial term of every minor of $\mathcal{X}^{\{2\}}$ and of $\mathcal{X}^{\{3\}}$ is its diagonal term. For example the lexicographic order induced by

is diagonal.

$$
x_{111}>x_{112}>x_{113}>x_{121}>\cdots>x_{1 a b}>x_{211}>\cdots>x_{2 a b}
$$

One can easily check that in this case the defining ideal of the Segre embedding of $\mathbb{P}^{1} \times \mathbb{P}^{a-1} \times \mathbb{P}^{b-1}$ in $\mathbb{P}^{2 a b-1}$, that is, the Hibi ideal associated to the poset $[2] \times[a] \times[b]$, is indeed the ideal generated by the 2 -minors of the second and third unfolding, that is,

$$
I(a, b)=I_{2}\left(\mathcal{X}^{\{2\}}\right)+I_{2}\left(\mathcal{X}^{\{3\}}\right) .
$$

For example, the Hibi relation $x_{213} x_{124}-x_{113} x_{224}$ is not a minor in $\mathcal{X}^{\{2\}}$ or $\mathcal{X}^{\{3\}}$ but can be written as a sum of a 2-minor of $\mathcal{X}^{\{2\}}$ and a 2-minor of $\mathcal{X}^{\{3\}}$ :

$$
x_{213} x_{124}-x_{113} x_{224}=\left(x_{213} x_{124}-x_{114} x_{223}\right)+\left(x_{114} x_{223}-x_{113} x_{224}\right) .
$$

We start by identifying a Gröbner basis for the ideal $I(a, b)$ itself.

Proposition 3.1. The 2-minors of the second and the third unfoldings of $\mathcal{X}$ are a Gröbner basis of $I(a, b)$ with respect to any diagonal term order.

Proof. It is not restrictive to consider the lexicographic order $\tau$ induced by $x_{111}>$ $x_{112}>x_{113}>x_{121}>\cdots>x_{1 a b}>x_{211}>\cdots>x_{2 a b}$. We first consider the following subsets of 2-minors in $I_{2}$ :

$$
\begin{aligned}
& M^{\{1\}}=\left\{x_{1 \alpha_{2} \alpha_{3}} x_{2 \beta_{2} \beta_{3}}-x_{1 \beta_{2} \alpha_{3}} x_{2 \alpha_{2} \beta_{3}}: \alpha_{2}<\beta_{2}\right\} \\
& M^{\{2\}}=\left\{x_{1 \alpha_{2} \alpha_{3}} x_{2 \beta_{2} \beta_{3}}-x_{1 \alpha_{2} \beta_{3}} x_{2 \beta_{2} \alpha_{3}}: \alpha_{3}<\beta_{3}\right\} \\
& M^{\{3\}}=\left\{x_{1 \alpha_{2} \alpha_{3}} x_{1 \beta_{2} \beta_{3}}-x_{1 \beta_{2} \alpha_{3}} x_{1 \alpha_{2} \beta_{3}}: \alpha_{2}<\beta_{2}, \alpha_{3}<\beta_{3}\right\} \\
& M^{\{4\}}=\left\{x_{2 \alpha_{2} \alpha_{3}} x_{2 \beta_{2} \beta_{3}}-x_{2 \beta_{2} \alpha_{3}} x_{2 \alpha_{2} \beta_{3}}: \alpha_{2}<\beta_{2}, \alpha_{3}<\beta_{3}\right\},
\end{aligned}
$$


and set $M=M^{\{1\}} \cup M^{\{2\}} \cup M^{\{3\}} \cup M^{\{4\}}$. Notice that $G^{\{2\}}=M^{\{1\}} \cup M^{\{3\}} \cup M^{\{4\}}$ is the set of the 2-minors of the second unfolding and that $G^{\{3\}}=M^{\{2\}} \cup M^{\{3\}} \cup M^{\{4\}}$ is the set of the 2-minors of the third unfolding.

It is well known and easy to check that $G^{\{2\}}$ and $G^{\{3\}}$ are Gröbner basis of the ideals they generate with respect to $\tau$. Hence it is enough to consider the $S$-polynomials $S(f, g)$ of $f, g$ with $f \in M^{\{1\}}$ and $g \in M^{\{2\}}$ whose initial monomials in $(S(f, g))$ are not relatively prime. There are two cases.

First let $f=x_{1 \alpha_{2} \alpha_{3}} x_{2 \beta_{2} \beta_{3}}-x_{1 \beta_{2} \alpha_{3}} x_{2 \alpha_{2} \beta_{3}}$ and $g=x_{1 \alpha_{2} \alpha_{3}} x_{2 \hat{\beta}_{2} \hat{\beta}_{3}}-x_{1 \alpha_{2} \hat{\beta}_{3}} x_{2 \hat{\beta}_{2} \alpha_{3}}$, with $\alpha_{2}<\beta_{2}$ and $\alpha_{3}<\hat{\beta}_{3}$. Thus $S(f, g)=-x_{1 \beta_{2} \alpha_{3}} x_{2 \alpha_{2} \beta_{3}} x_{2 \hat{\beta}_{2} \hat{\beta}_{3}}+x_{1 \alpha_{2} \hat{\beta}_{3}} x_{2 \hat{\beta}_{2} \alpha_{3}} x_{2 \beta_{2} \beta_{3}}$, and since $\alpha_{2}<\beta_{2}$, one has in $(S(f, g))=x_{1 \alpha_{2} \hat{\beta}_{3}} x_{2 \beta_{2} \beta_{3}} x_{2 \hat{\beta}_{2} \alpha_{3}}$. Dividing $S(f, g)$ by $h_{0}=x_{1 \alpha_{2} \hat{\beta}_{3}} x_{2 \beta_{2} \beta_{3}}-x_{1 \beta_{2} \hat{\beta}_{3}} x_{2 \alpha_{2} \beta_{3}} \in M^{\{1\}}$ one gets

$$
S(f, g)=x_{2 \hat{\beta}_{2} \alpha_{3}} \cdot h_{0}-x_{2 \alpha_{2} \beta_{3}}\left(x_{1 \beta_{2} \alpha_{3}} x_{2 \hat{\beta}_{2} \hat{\beta}_{3}}-x_{1 \beta_{2} \hat{\beta}_{3}} x_{2 \hat{\beta}_{2} \alpha_{3}}\right)
$$

which reduces to zero modulo $M$ since $x_{1 \beta_{2} \alpha_{3}} x_{2 \hat{\beta}_{2} \hat{\beta}_{3}}-x_{1 \beta_{2} \hat{\beta}_{3}} x_{2 \hat{\beta}_{2} \alpha_{3}} \in M^{\{2\}}$.

The second case is $f=x_{1 \alpha_{2} \alpha_{3}} x_{2 \beta_{2} \beta_{3}}-x_{1 \beta_{2} \alpha_{3}} x_{2 \alpha_{2} \beta_{3}}$ and $g=x_{1 \hat{\alpha}_{2} \hat{\alpha}_{3}} x_{2 \beta_{2} \beta_{3}}-$ $x_{1 \hat{\alpha}_{2} \beta_{3}} x_{2 \beta_{2} \hat{\alpha}_{3}}$, with $\alpha_{2}<\beta_{2}$ and $\hat{\alpha}_{3}<\beta_{3}$. Thus $S(f, g)=-x_{1 \hat{\alpha}_{2} \hat{\alpha}_{3}} x_{1 \beta_{2} \alpha_{3}} x_{2 \alpha_{2} \beta_{3}}+$ $x_{1 \alpha_{2} \alpha_{3}} x_{1 \hat{\alpha}_{2} \beta_{3}} x_{2 \beta_{2} \hat{\alpha}_{3}}$.

If $\hat{\alpha}_{2}>\alpha_{2}$, then $\operatorname{in}(S(f, g))=x_{1 \alpha_{2} \alpha_{3}} x_{1 \hat{\alpha}_{2} \beta_{3}} x_{2 \beta_{2} \hat{\alpha}_{3}}$ and dividing by $h_{1}=$ $x_{1 \alpha_{2} \alpha_{3}} x_{2 \beta_{2} \hat{\alpha}_{3}}-x_{1 \beta_{2} \alpha_{3}} x_{2 \alpha_{2} \hat{\alpha}_{3}} \in M^{\{1\}}$ leads to

$$
S(f, g)=x_{1 \hat{\alpha}_{2} \beta_{3}} \cdot h_{1}-x_{1 \beta_{2} \alpha_{3}}\left(x_{1 \hat{\alpha}_{2} \hat{\alpha}_{3}} x_{2 \alpha_{2} \beta_{3}}-x_{1 \hat{\alpha}_{2} \beta_{3}} x_{2 \alpha_{2} \hat{\alpha}_{3}}\right)
$$

which reduces to zero modulo $M$ since $x_{1 \hat{\alpha}_{2} \hat{\alpha}_{3}} x_{2 \alpha_{2} \beta_{3}}-x_{1 \hat{\alpha}_{2} \beta_{3}} x_{2 \alpha_{2} \hat{\alpha}_{3}} \in M^{\{2\}}$.

To conclude we have to consider three more situations, and arguing as before.

If $\hat{\alpha}_{2}<\alpha_{2}$, then $\operatorname{in}(S(f, g))=x_{1 \hat{\alpha}_{2} \hat{\alpha}_{3}} x_{1 \beta_{2} \alpha_{3}} x_{2 \alpha_{2} \beta_{3}}$ and we get $S\left(f_{2}, g_{2}\right)=$ $x_{1 \hat{\alpha}_{2} \beta_{3}} \cdot h_{4}-x_{1 \beta_{2} \alpha_{3}} h_{3}$ with $h_{3}=x_{1 \hat{\alpha}_{2} \hat{\alpha}_{3}} x_{2 \alpha_{2} \beta_{3}}-x_{1 \hat{\alpha}_{2} \beta_{3}} x_{2 \alpha_{2} \hat{\alpha}_{3}} \in M^{\{2\}}$ and $h_{4}=-x_{1 \beta_{2} \alpha_{3}} x_{2 \alpha_{2} \hat{\alpha}_{3}}+x_{1 \alpha_{2} \alpha_{3}} x_{2 \beta_{2} \hat{\alpha}_{3}} \in M^{\{1\}}$.

The case $\hat{\alpha}_{2}=\alpha_{2}$ and $\hat{\alpha}_{3}<\alpha_{3}$ works as the previous one.

If $\hat{\alpha}_{2}=\alpha_{2}$ and $\hat{\alpha}_{3}=\alpha_{3}$, then $\operatorname{in}(S(f, g))=x_{1 \alpha_{2} \alpha_{3}} x_{1 \alpha_{2} \beta_{3}} x_{2 \beta_{2} \alpha_{3}}$ and one has $S(f, g)=x_{1 \alpha_{2} \alpha_{3}}\left(h_{5}-h_{6}\right)$, where $h_{5}=x_{1 \alpha_{2} \beta_{3}} x_{2 \beta_{2} \alpha_{3}}-x_{1 \beta_{2} \beta_{3}} x_{2 \alpha_{2} \alpha_{3}} \in M^{\{1\}}$ and $h_{6}=x_{1 \beta_{2} \alpha_{3}} x_{2 \alpha_{2} \beta_{3}}-x_{1 \beta_{2} \beta_{3}} x_{2 \alpha_{2} \alpha_{3}} \in M^{\{2\}}$.

Finally, if $\alpha_{2}=\hat{\alpha_{2}}$ and $\alpha_{3}<\hat{\alpha}_{3}$, then $\operatorname{in}(S(f, g))=x_{1 \alpha_{2} \alpha_{3}} x_{1 \alpha_{2} \beta_{3}} x_{2 \beta_{2} \hat{\alpha}_{3}}$ and we have $S(f, g)=x_{1 \alpha_{2} \beta_{3}} \cdot h_{7}-x_{1 \beta_{2} \alpha_{3}} \cdot h_{8}$ where $h_{7}=x_{1 \alpha_{2} \alpha_{3}} x_{2 \beta_{2} \hat{\alpha}_{3}}-x_{1 \beta_{2} \alpha_{3}} x_{2 \alpha_{2} \hat{\alpha}_{3}} \in M^{\{1\}}$ and $h_{8}=x_{1 \alpha_{2} \hat{\alpha}_{3}} x_{2 \alpha_{2} \beta_{3}}-x_{1 \alpha_{2} \beta_{3}} x_{2 \alpha_{2} \hat{\alpha}_{3}} \in M^{\{2\}}$.

Thus in these three situations $S(f, g)$ reduces to zero modulo $M$; this finishes the proof.

As we have seen in Example 1.1 instead of looking at the second and the third unfolding of the tensor $\mathcal{X}$ separately, we can combine them into a single arrangement:

$$
W=\begin{aligned}
& X Y \\
& Y
\end{aligned}
$$

where $X=\left(x_{1 i j}\right)$ and $Y=\left(x_{2 i j}\right)$ are both of dimension $a \times b$.

Thus $I(a, b)$ is generated by the 2 -minors in the arrangement and the initial ideal $\operatorname{in}(I(a, b))$ is generated by the 2-diagonals in the arrangement. We introduce a partial order in the set of variables so that the 2-diagonals are exactly the pairs of comparable elements. To this end, we identify the set of the variables with:

$$
P=\{(i, j): 1 \leqslant i \leqslant a, 1 \leqslant j \leqslant 2 b\}
$$

associating $x_{1 i j}$ to $(i, j)$ and $x_{2 i j}$ to $(i, b+j)$. 
In $P$ we introduce the following partial order:

$$
(x, y) \preceq(z, t) \text { if and only if }\left\{\begin{array}{l}
(x, y)=(z, t) \text { or } \\
x<z, y<t \text { or } \\
y \leqslant b, t \geqslant b+1, y<t-b .
\end{array}\right.
$$

With this notation, the generators of in $(I(a, b))$ are exactly the pairs of distinct comparable elements in $P$. Now, in $(I(a, b))$, being a square-free monomial ideal, corresponds to a simplicial complex that we denote by $\Delta_{2}$. Here we use the index 2 to recall the generators of the associated ideal are the 2-diagonals, that is, the pairs of comparable elements in $P$.

Recall that an antichain in the partially ordered set $P$ is a set of elements no two of which are comparable to each other. Therefore the elements of $\Delta_{2}$ are the antichains of $P$. Moreover a saturated (or maximal) antichain of $P$ is an antichain that is, maximal with respect to inclusions. Therefore the saturated antichains of $P$ are the facets of $P$.

A subset $\left\{\left(h_{1}, k_{1}\right), \ldots,\left(h_{s}, k_{s}\right)\right\}$ of elements of $P$ is said to be a path with starting point $\left(h_{1}, k_{1}\right)$ and ending point $\left(h_{s}, k_{s}\right)$ if $\left(h_{t+1}, k_{t+1}\right)-\left(h_{t}, k_{t}\right) \in\{(1,0),(0,-1)\}$ for all $t=1, \ldots, s-1$. We will represent $P$ with the matrix orientation, that is, with $(1,1)$ in the top left corner and $(a, 2 b)$ in the bottom right corner. With this representation a path consists of a sequence of steps to the left and steps down.

THEOREM 3.2. The facets of $\Delta_{2}$ are the paths starting in $(1, b+h)$ and ending in $(a, h)$ for some $h$ with $1 \leqslant h \leqslant b$.

Proof. Let $F$ be a path in $P$ starting in $(1, b+h)$ and ending in $(a, h)$, with $1 \leqslant h \leqslant b$, and passing through $(k, b+1)$ and $(k, b)$. We first prove that $F$ is a facet. It is clear that no pair of points in $F$ is comparable, thus $F \in \Delta_{2}$. Since we know that $\Delta_{2}$ is the simplicial complex associated to in $(I(a, b))$ and $R / I(a, b)$ has dimension $a+b$, it follows that the facets of $\Delta_{2}$ have at most cardinality $a+b$. Hence $F$ is a facet of $\Delta_{2}$.

To conclude we show that every facet in $\Delta_{2}$ is a subset of a path $F$ as described above. Let $E \subset P$ be in $\Delta_{2}$, that is, $E$ does not contain any pair of comparable elements. Note that by the first condition of $\preceq$ we have that $E$ is an antichain, so it is of the form

$$
E=\left\{\left(i_{1}, j_{1}\right),\left(i_{2}, j_{2}\right), \ldots,\left(i_{\alpha}, j_{\alpha}\right),\left(h_{1}, k_{1}\right),\left(h_{2}, k_{2}\right), \ldots,\left(h_{\beta}, k_{\beta}\right)\right\}
$$

with $i_{1} \geqslant \cdots \geqslant i_{\alpha} \geqslant h_{1} \geqslant \cdots \geqslant h_{\beta}$ and $j_{1} \leqslant \cdots \leqslant j_{\alpha} \leqslant b<b+1 \leqslant k_{1} \leqslant \cdots \leqslant k_{\beta}$. Moreover we can assume that $j_{t+1}>j_{t}$ whenever $i_{t+1}=j_{t}$, and $k_{t+1}>k_{t}$ whenever $h_{t+1}=h_{t}$. We prove that $E$ is contained in a path $F$ starting in $\left(1, b+j_{1}\right)$ and ending in $\left(a, j_{1}\right)$.

If $\beta=0$, that is, $E=\left\{\left(i_{1}, j_{1}\right),\left(i_{2}, j_{2}\right), \ldots,\left(i_{\alpha}, j_{\alpha}\right)\right\}$, then we can saturate the antichain, and add the points $\left(i_{1}+1, j_{1}\right),\left(i_{1}+2, j_{1}\right), \ldots,\left(a, j_{1}\right)$ and the points $\left(i_{\alpha}-\right.$ $\left.1, j_{\alpha}\right),\left(i_{\alpha}-2, j_{\alpha}\right), \ldots,\left(1, j_{\alpha}\right),\left(1, j_{\alpha}+1\right),\left(1, j_{\alpha}+2\right), \ldots,\left(1, b+j_{1}\right)$, so that we obtain the path $F$. Analogously one concludes if $\alpha=0$, that is, if

$$
E=\left\{\left(h_{1}, k_{1}\right),\left(h_{2}, k_{2}\right), \ldots,\left(h_{\beta}, k_{\beta}\right)\right\} .
$$

Suppose now that both $\alpha$ and $\beta$ are not zero. Then $k_{\beta} \leqslant b+j_{1}$, otherwise it would be $j_{1}<k_{\beta}-b$, thus $\left(i_{1}, j_{1}\right) \prec\left(h_{\beta}, k_{\beta}\right)$, which contradicts $E \in \Delta_{2}$. So we can saturate and add the points $\left(i_{1}+1, j_{1}\right),\left(i_{1}+2, j_{1}\right), \ldots,\left(a, j_{1}\right)$ and the points $\left(h_{\beta}-1, k_{\beta}\right),\left(h_{\beta}-2, k_{\beta}\right),\left(1, k_{\beta}\right),\left(1, k_{\beta}+1\right), \ldots,\left(1, b+j_{1}\right)$, so obtaining also in this case the facet $F$ containing $E$. This finishes the proof.

REMARK 3.3. Let $F$ be a path in $P$, starting in $(1, b+h)$ and ending in $(a, h)$, with $1 \leqslant h \leqslant b$, and passing through $(k, b+1)$ and $(k, b)$. It is clear that $F$ corresponds 
to a path $F^{\prime}$ in $P^{\prime}=\{(i, j): 1 \leqslant i \leqslant a, 1 \leqslant j \leqslant 2 b$ or $a+1 \leqslant i \leqslant 2 a, 1 \leqslant j \leqslant b\}$ starting in $(1, b+h)$ and ending in $(a+k, 1)$, with $k \leqslant a$, and passing through $(k, b+1)$, $(k, b)$, and $(a, h)$ (see Figure 1$)$. Thus one can also rephrase the theorem in term of paths in $P^{\prime}$. In the following we will use both the descriptions of $\Delta_{2}$, as subset of $P$ and of $P^{\prime}$.
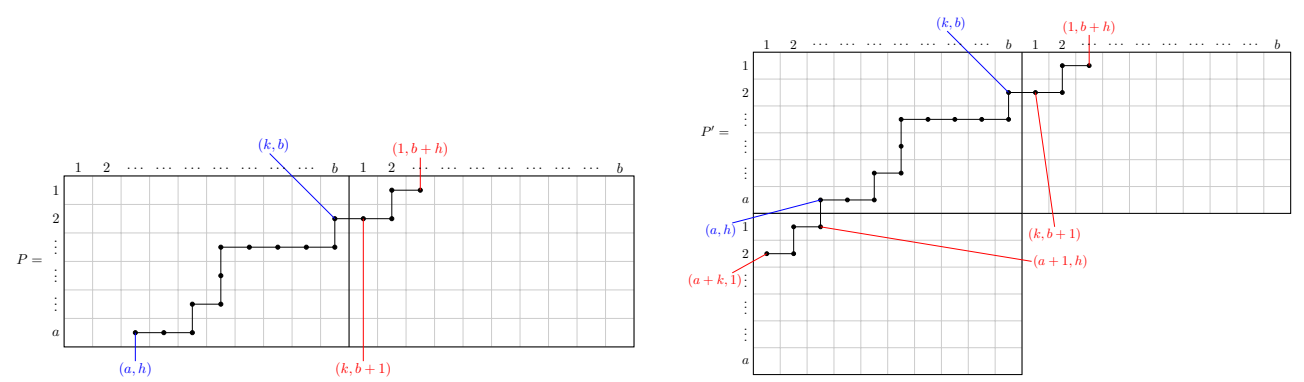

Figure 1. An example of a path in $P$ and the corresponding path in $P^{\prime}$.

\section{SECANT IDEALS}

Our goal is to prove:

THEOREM 4.1. Let $K$ be a field of arbitrary characteristic and $a, b$ positive integers. Then

(1) the defining ideal $I(a, b)^{\{t\}}$ of the $t$-secant variety $\sigma_{t}(2, a, b)$ of the Segre variety $\operatorname{Seg}(2, a, b)$ is

$$
I_{t+1}\left(\mathcal{X}^{\{2\}}\right)+I_{t+1}\left(\mathcal{X}^{\{3\}}\right)
$$

(2) the $(t+1)$-minors of $\mathcal{X}^{\{2\}}$ and $\mathcal{X}^{\{3\}}$ are a Gröbner basis of $I(a, b)^{\{t\}}$ with respect to any diagonal term order.

(3) $R / I(a, b)^{\{t\}}$ is a Cohen-Macaulay domain.

To simplify the notation in the following we will denote by $I_{t+1}$ the ideal $I_{t+1}\left(\mathcal{X}^{\{2\}}\right)+I_{t+1}\left(\mathcal{X}^{\{3\}}\right)$.

The ideal $I(a, b)$ defines $\operatorname{Seg}(2, a, b)$ and is generated by the 2-minors of the arrangement $W$, that is, $I_{2}=I(a, b)$. Hence the secant variety $\sigma_{t}(2, a, b)$ is contained in the variety of tensors whose second and third unfolding are of rank at most $t$. Hence it follows that:

LEMMA 4.2. The ideal $I_{t+1}$ is contained in $I_{2}^{\{t\}}$.

REMARK 4.3. Mirsky's theorem [6] states that the size of the largest chain in a finite poset equals the smallest number of antichains into which the poset may be partitioned. The simplical complex $\Delta_{2}^{\{t\}}$ consists of the subsets of $P$ that can be decomposed into the union of $t$ antichains. According to Mirsky's theorem, they are exactly the subsets of $P$ that do not contains chains of $t+1$ elements. Equivalently the ideal of $\Delta_{2}^{\{t\}}$ is generated by the chains of $t+1$ elements, that is, the leading terms of the $(t+1)$-minors of $W$.

Proof of Theorem 4.1 parts (1) and (2). Denote by $J_{t}$ the ideal generated by the initial terms of the $(t+1)$-minors of the arrangement $W$, that is, the monomials correponding to the $t+1$-diagonals of $P^{\prime}$. Notice that we have the following relations

$$
J_{t} \subseteq \operatorname{in}\left(I_{t+1}\right) \underset{4.2}{\subseteq} \operatorname{in}\left(I_{2}^{\{t\}}\right) \underset{2.3}{\subseteq} \operatorname{in}\left(I_{2}\right)^{\{t\}}=I_{\Delta_{2}}^{\{t\}}=I_{\Delta_{2}^{\{t\}}}=J_{t},
$$


where the last equality follows from Remark 4.3. It follows that $J_{t}=\operatorname{in}\left(I_{t+1}\right)$ and $I(a, b)^{\{t\}}=I_{t+1}$. This concludes the proof.

Note that all the inclusions in Eq. (1) are indeed equalities. Thus one has:

COROLlary 4.4. The diagonal term orders are delightful for the ideal $I_{2}$.

To prove 4.1 part (3) we need to describe better the facets of the simplicial complex $\Delta_{t+1}$ associated to in $\left(I(a, b)^{\{t\}}\right)$. As a special case of Theorem $4.1(2)$ we have that if $t \geqslant \min (2 a, b)$ the ideal $I(a, b)^{\{t\}}$ is trivial and if $a \leqslant t<\min (2 a, b)$ then $I(a, b)^{\{t\}}$ is a generic determinantal ideal and hence well understood. So in the remaining part of the paper we will assume that $t<a$.

We recall this well-known result.

THEOREM 4.5 ([3, Theorem 1]). Let $P$ be a prime ideal in a polynomial ring $R$, and let $\prec$ be any term order on $R$. Then the simplicial complex associated to $\operatorname{in}_{\prec}(P)$ is pure of dimension $\operatorname{dim}(R / P)-1$ and strongly connected.

Denote by $\mathcal{F}(\Delta)$ the set of the facets of a simplicial complex $\Delta$.

Lemma 4.6. The ideal $I_{t+1}$ is prime. Moreover the simplicial complex $\Delta_{t+1}$ is pure of dimension $\operatorname{dim} \Delta_{t+1}=(a+b) t-1$, and

$$
\mathcal{F}\left(\Delta_{t+1}\right)=\left\{F_{1} \cup F_{2} \cup \cdots \cup F_{t}: F_{i} \in \mathcal{F}\left(\Delta_{2}\right) \text { and } F_{i} \cap F_{j}=\varnothing \text { for } i \neq j\right\} .
$$

Proof. The ideal $I(a, b)$ is a prime and so is its secant ideal $I_{t+1}$. Thus $\Delta_{t+1}$ is pure, since by Corollary 4.4 it is equal to $\Delta_{2}^{\{t\}}$, which is pure by Theorem 4.5. Since any face of $\Delta_{t+1}$ is the union of $t$ faces of $\Delta_{2}$ and the facets $\Delta_{2}$ have $a+b$ elements, to conclude it is enough to exhibit $t$ facets, say $F_{1}, \ldots, F_{t}$, of $\Delta_{2}$ that are pairwise disjoint. Let $F_{1}, \ldots, F_{t} \in \mathcal{F}\left(\Delta_{2}\right)$ be given as follows. Firstly $F_{1}$ is the "right most" path in $P$ from $(1,2 b)$ to $(a, b)$, that is,

$$
\{(1,2 b), \ldots,(a-1,2 b),(a, 2 b), \ldots,(a, b+2)(a, b+1),(a, b)\} .
$$

Secondly $F_{2}$ is the "right most" path in $P$ from $(1,2 b-1)$ to $(a, b-1)$ that does not intersect $F_{1}$ and for $i=3, \ldots, t, F_{i}$ is the "right most" path in $P$ from $(1,2 b-i+1)$ to $(a, b-i+1)$ to that does not intersect $F_{i-1}$. For example the picture 2 shows this construction in the extremal case $t=a=6, b=10$.

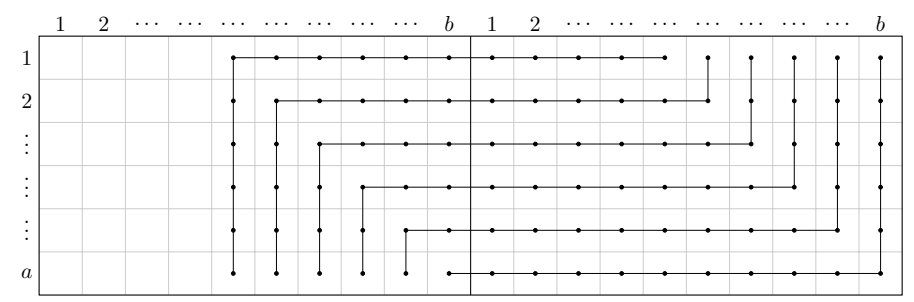

Figure 2. A facet in $\Delta_{7}$ with $a=6, b=10$

In the following we say that an element $(x, y)$ of a path $G$ in $P$ is a low-right corner of $G$ if also $(x-1, y)$ and $(x, y-1)$ are in $G$. By using the description of the facets of $\Delta_{t+1}$ given above we prove the following.

Proposition 4.7. The simplicial complex $\Delta_{t+1}$ is shellable. 
Proof. Given $x=(u, v) \in P$, we set $\mathcal{R}_{x}=\{(i, j) \in P: i>u, j>v\}, \overline{\mathcal{R}}_{x}=\{(i, j) \in$ $P: i \geqslant u, j \geqslant v\}, \mathcal{L}_{x}=\{(i, j) \in P: i<u, j<v\}$, and $\overline{\mathcal{L}}_{x}=\{(i, j) \in P: i \leqslant u, j \leqslant$ $v\}$. Furthermore, if $G \subseteq P$, we let $\mathcal{R}_{G}=\bigcup_{x \in G} \mathcal{R}_{x}, \overline{\mathcal{R}}_{G}=\bigcup_{x \in G} \overline{\mathcal{R}}_{x}, \mathcal{L}_{G}=\bigcup_{x \in G} \mathcal{L}_{x}$ and $\overline{\mathcal{L}}_{G}=\bigcup_{x \in G} \mathcal{L}_{x}$. Finally, we let $x^{L}=(u-1, v-1)$.

First we define a partial order on the set of the facets of $\Delta_{t+1}$. Let $F=G_{1} \cup \cdots \cup G_{t}$ and $F^{\prime}=G_{1}^{\prime} \cup \cdots \cup G_{t}^{\prime}$ be two facets of $\Delta_{t+1}$, where $G_{i}$ is a path starting in $\left(1, b+h_{i}\right)$ and ending in $\left(a, h_{i}\right)$ and $G_{i}^{\prime}$ is a path starting in $\left(1, b+k_{i}\right)$ and ending in $\left(a, k_{i}\right)$. Assume that $h_{1}<\cdots<h_{t}$ and $k_{1}<\cdots<k_{t}$. Set

$$
F \preceq F^{\prime} \quad \text { if and only if } \quad G_{i}^{\prime} \subseteq \overline{\mathcal{R}}_{G_{i}} \text { for all } i=1, \ldots, t .
$$

Extend $\preceq$ to a total order $\leqslant$ on the set of the facets of $\Delta_{t+1}$. To prove that $\Delta_{t+1}$ is shellable, we need to show that, given any two facets $F^{\prime}<F$, there exists $x \in F \backslash F^{\prime}$ and a facet $F^{\prime \prime}$ such that $F^{\prime \prime}<F$ and $F \backslash F^{\prime \prime}=\{x\}$.

Let $F^{\prime}<F$ be two given facets decomposed as above. Since $F \npreceq F^{\prime}$, we may consider the least integer $i$ such that $G_{i}^{\prime} \nsubseteq \overline{\mathcal{R}}_{G_{i}}$, with $i \leqslant t$. Thus there exists $y \in G_{i}^{\prime}$ such that $y \notin \overline{\mathcal{R}}_{G_{i}}$, that is, $y \in \mathcal{L}_{G_{i}}$. Choose such a $y$ with the smallest row index possible, and note that $\mathcal{R}_{y} \cap G_{i} \neq \varnothing$.

Now we consider two cases, according to the existence of a low-right corner $x$ of $G_{i}$ which is in $\mathcal{R}_{y}$, cf. Figure 3 .

CASE 1. Assume that such a corner does not exist. Let $y=(r, s)$; note that since $y \in \mathcal{L}_{G_{i}}$, then there exist $x^{\prime} \in G_{i}$ such that $y \in \mathcal{L}_{x^{\prime}}$, and by assumption $x^{\prime}=\left(r^{\prime}, k_{i}\right)$. In particular $k_{i} \leqslant s<h_{i}$, thus $k_{i}<h_{i}$. Note that $\left(1, b+h_{i}-1\right)$ is in $G_{i}$, because otherwise we would have $\left(2, b+h_{i}\right) \in G_{i}$ and thus $y=\left(1, b+k_{i}\right)$, and there would be a low-right corner of $G_{i}$ belonging to $\mathcal{R}_{y}$, a contradiction. Moreover $\left(1, b+h_{i}\right)$ is not in $G_{i}^{\prime}$.

Now if $i=t$ or if $i<t$ and $\left(1, b+h_{i}\right) \notin G_{i+1}^{\prime}$, then $\left(1, b+h_{i}\right) \in F \backslash F^{\prime}$ and $F^{\prime \prime}=\left(F \cup\left\{\left(a, h_{i}-1\right)\right\}\right) \backslash\left\{\left(1, b+h_{i}\right)\right\}$ is facet such that $F^{\prime \prime} \preceq F$, thus $F^{\prime \prime}<F$, as desired. Otherwise, if $i<t$ and $\left(1, b+h_{i}\right) \in G_{i+1}^{\prime}$, then $h_{i} \leqslant k_{i+1}$, and there exists an element $y^{\prime} \in G_{i+1}^{\prime}$ such that $y^{\prime} \notin \overline{\mathcal{R}}_{G_{i+1}}$. So we can start again arguing on $y^{\prime}$ as we did on $y$, by using Case 1 or Case 2 .

CASE 2. Suppose that such a corner exists. Since $y \in G_{i}^{\prime}$ and the path of $F^{\prime}$ are disjoint, one has that $y \in \mathcal{R}_{G_{i-1}^{\prime}}$. By the minimality of $i, G_{i-1}^{\prime} \subseteq \overline{\mathcal{R}}_{G_{i-1}}$, thus $y \in \mathcal{R}_{G_{i-1}}$. Since $y \in \mathcal{L}_{x}$, it follows that $x^{L} \in \mathcal{R}_{G_{i-1}}$ and, consequently, $x^{L} \notin G_{i-1}$, thus $x^{L} \notin F$.

Now if $x \notin F^{\prime}$, then $F^{\prime \prime}=\left(F \cup\left\{x^{L}\right\}\right) \backslash\{x\}$ is a facet of $\Delta_{t}$ such that $F^{\prime \prime} \preceq F$, thus $F^{\prime \prime}<F$, as desired.

It remains to consider the case in which $x \in F^{\prime}$, that is, $i<t$ and $x \in G_{i+1}^{\prime}$. Since $x \notin \overline{\mathcal{R}}_{G_{i+1}}$, we can start again applying to $x$ the arguments we have used for $y$, following Case 1 or Case 2 .

It is clear that this procedure concludes after a finite number of steps, proving that $\Delta_{t+1}$ is shellable.

As a consequence we can conclude the proof of Theorem 4.1.

Proof of 4.1 part (3). Since $\Delta_{t+1}$ is shellable the associate Stanley-Reisner ring $R / I_{\Delta_{t+1}}$ is Cohen-Macaulay [1, Theorem 5.1.13]. Since $\operatorname{in}\left(I(a, b)^{\{t\}}\right)=I_{\Delta_{t+1}}$ it follows that $R / I(a, b)^{\{t\}}$ is Cohen-Macaulay as well.

THEOREM 4.8. With the notation of 4.1 , the ring $R / I(a, b)^{\{t\}}$, with $t \leqslant a-1$, is a domain of dimension $(a+b) t$, and of multiplicity

$$
\sum_{1 \leqslant h_{1}<\cdots<h_{t} \leqslant b} \operatorname{det}\left(g_{i j}\right)
$$




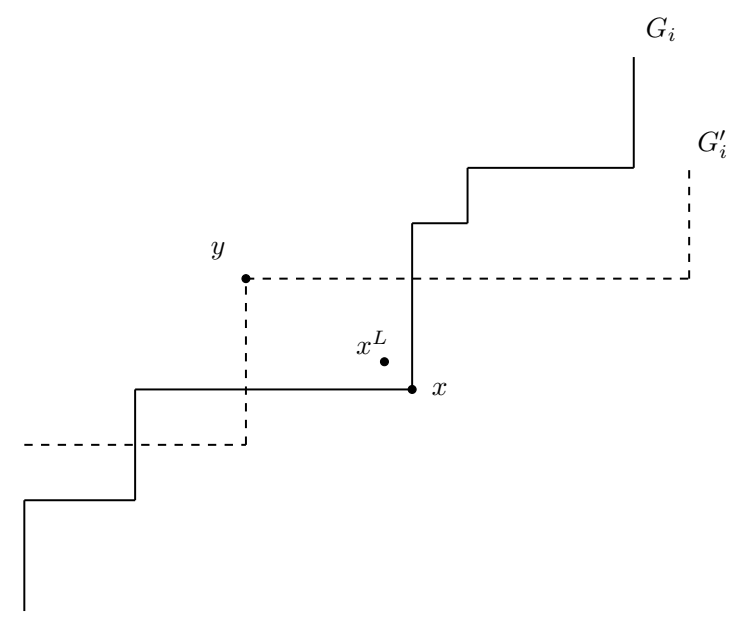

FIGURE 3 .

with $g_{i j}=\left(\begin{array}{c}a+b-h_{j}+h_{i}-1 \\ a-1\end{array}\right)$ for $1 \leqslant i, j \leqslant t$. Furthermore the Castelnuovo Mumford regularity of $R / I(a, b)^{\{t\}}$ is less than or equal to at, with equality if $b \geqslant 2 t$.

Proof. Since $\Delta_{t+1}$ is pure, the multiplicity of $R / I(a, b)^{\{t\}}$ is equal to the number of the facets of $\Delta_{t+1}$. The set of the facets of $\Delta_{t+1}$ can be written as the disjoint union of the set of non-intersecting paths with starting points $Q_{1}=\left(1, b+h_{1}\right), \ldots, Q_{t}=\left(1, b+h_{t}\right)$ and ending points $P_{1}=\left(a, h_{1}\right), \ldots, P_{t}=\left(a, h_{t}\right)$ where $1 \leqslant h_{1}<\cdots<h_{t} \leqslant b$. By a well known formula of Gessel-Viennot [2], the number of non-intersecting paths with starting points $Q_{1}, \ldots, Q_{t}$ and ending points $P_{1}, \ldots, P_{t}$ is the determinant of the matrix whose $(i, j)$-th entry is the number of paths from $Q_{i}$ to $P_{j}$ which is easily seen to be equal to

$$
\left(\begin{array}{c}
a+b-h_{j}+h_{i}-1 \\
a-1
\end{array}\right) .
$$

This gives the formula for the multiplicity.

Given a shellable simplical complex $\Delta$ with a shelling $G_{1}, \ldots, G_{v}$ we set

$$
r\left(G_{i}\right)=\left\{x \in G_{i}: \text { there exists } j<i \text { such that } G_{i} \backslash G_{j}=\{x\}\right\} .
$$

If $K[\Delta]$ is the Stanley-Reisner ring associated to $\Delta$, then one has

$$
\operatorname{reg}(K[\Delta])=\max \left\{\left|r\left(G_{i}\right)\right|: i=1, \ldots, v\right\} .
$$

Applying this to $\Delta_{t+1}$ and using the fact that $R / I(a, b)^{\{t\}}$ and $K\left[\Delta_{t+1}\right]$ have the same regularity, we have

$$
\operatorname{reg}(R / I(a, b))=\max \left\{|r(G)|: G \text { is a facet of } \Delta_{t+1}\right\} .
$$

For a facet $G=\bigcup_{i=1}^{t} F_{i}$ and with respect to the shelling described in Proposition 4.7 one has that

$$
r(G) \subseteq \bigcup_{i=1}^{t}\left\{x \in F_{i}: x \text { is a right turn of } F_{i} \text { or } x \text { is the starting point of } F_{i}\right\} .
$$

Since any path $F_{i}$ has at most $a-1$ such turns, we have that $|r(G)| \leqslant a t$ and hence $\operatorname{reg}\left(R / I(a, b)^{\{t\}}\right) \leqslant a t$. If $b \geqslant 2 t$ then one can actually find a facet $G$ of $\Delta_{t+1}$ such that $|r(G)|=a t$ proving that for $b \geqslant 2 t$ one has $\operatorname{reg}\left(R / I(a, b)^{\{t\}}\right)=a t$. The construction of the facet $G$ such that $|r(G)|=a t$ is illustrated by the following example, where 
$(a, b)=(5,8), t=4$, and a facet $G$ is represented with a dot in every point belonging to $r(G)$ :

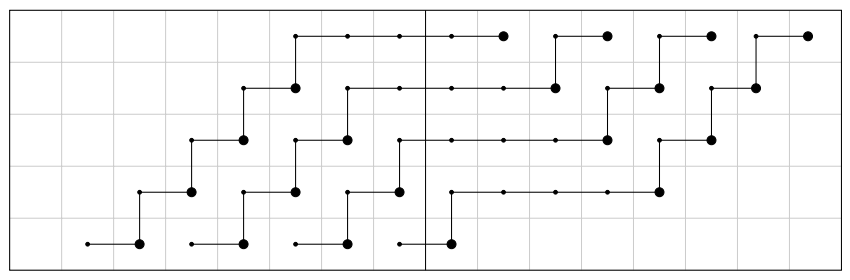

In the case $a=b=2 t$ the facet with $|r(G)|=a t$ described in the proof is indeed the only facet of $\Delta_{t+1}$ with that property, as in this example, with $(a, b)=(6,6)$, $t=3$ :

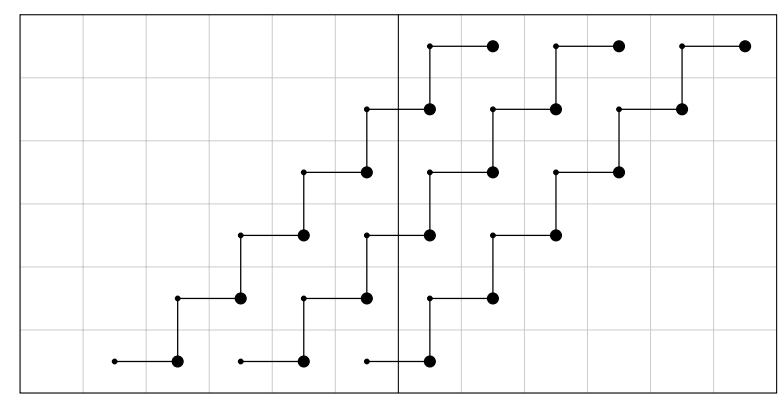

This implies that the highest non-zero entry of the $h$-vector of $\Delta_{t+1}$ is 1 . The latter is a necessary condition for the $R / I(a, b)^{\{t\}}$ to be Gorenstein. This leads to the following conjecture:

Conjecture 4.9. For any the ring $R / I(2 t, 2 t)^{\{t\}}$ is Gorenstein.

For $t \leqslant 3$ one can actually compute the numerator of the Hilbert series of $R / I(2 t, 2 t)^{\{t\}}$ and check that it is symmetric and hence verify, in view of [1, Corollary 4.4.6], that the conjecture holds. For example for $t=3$ the numerator of the Hilbert series of $R / I(6,6)^{\{3\}}$ turns out to be:

$$
\begin{array}{rl}
1+36 x+666 x^{2}+8 & 436 x^{3}+68526 x^{4}+366660 x^{5}+1330644 x^{6}+3296124 x^{7} \\
+5650866 x^{8}+ & 6762316 x^{9}+5650866 x^{10}+3296124 x^{11}+1330644 x^{12} \\
+ & 366660 x^{13}+68526 x^{14}+8436 x^{15}+666 x^{16}+36 x^{17}+x^{18} .
\end{array}
$$

\section{REFERENCES}

[1] Winfried Bruns and Jürgen Herzog, Cohen-Macaulay rings, Cambridge Studies in Advanced Mathematics, vol. 39, Cambridge University Press, Cambridge, 1993.

[2] Ira Gessel and Gérard Viennot, Binomial determinants, paths, and hook length formulae, Adv. Math. 58 (1985), no. 3, 300-321.

[3] Michael Kalkbrener and Bernd Sturmfels, Initial complexes of prime ideals, Adv. Math. 116 (1995), no. 2, 365-376.

[4] Joseph M. Landsberg, Tensors: geometry and applications, Graduate Studies in Mathematics, vol. 128, American Mathematical Society, Providence, RI, 2012.

[5] Joseph M. Landsberg and Jerzy Weyman, On the ideals and singularities of secant varieties of Segre varieties, Bull. Lond. Math. Soc. 39 (2007), no. 4, 685-697.

[6] Leon Mirsky, A dual of Dilworth's decomposition theorem, Amer. Math. Monthly 78 (1971), $876-877$.

[7] Luke Oeding, Are all secant varieties of Segre products arithmetically Cohen-Macaulay?, https: //arxiv.org/abs/1603.08980, 2016.

[8] Aron Simis and Bernd Ulrich, On the ideal of an embedded join, J. Algebra 226 (2000), no. 1, $1-14$. 
[9] Bernd Sturmfels and Seth Sullivant, Combinatorial secant varieties, Pure Appl. Math. Q. 2 (2006), no. 3, 867-891, Special Issue: In honor of Robert D. MacPherson. Part 1.

Aldo Conca, Dipartimento di Matematica, Università di Genova, via Dodecaneso 35, Genova, Italy

E-mail : conca@dima.unige.it

Emanuela De Negri, Dipartimento di Matematica, Università di Genova, via Dodecaneso 35, Genova, Italy

E-mail : denegri@dima.unige.it

Želuka Stojanac, Institute for Theoretical Physics, University of Cologne, Germany

E-mail : zeljka.stojanac@gmail.com 